\title{
Design of potent Mincle signalling agonists based on an alkyl $\beta$ - glucoside template
}

Received 00th January 20xx, Accepted 00th January 20xx

\author{
Dylan G.M. Smith, ${ }^{a}$ Yuki Hosono, ${ }^{b, c}$ Masahiro Nagata, ${ }^{b, c}$ Sho Yamasaki ${ }^{b, c}$ and Spencer J. Williams ${ }^{* a}$
}

DOI: $10.1039 / \times 0 x \times 00000 x$

The innate immune receptor Mincle senses lipid-based molecules derived from pathogens, commensals and altered self. Based on emerging structure-activity relationships we design simple alkyl 6$O$-acyl- $\beta$-D-glucosides that are effective agonists of Mincle and signal with potency on par with the prototypical ligand trehalose dimycolate.

Macrophage inducible C-type lectin (Mincle) receptor is an FcR $\gamma$-associated pattern recognition receptor involved in innate immunity. ${ }^{1}$ Mincle senses a range of self and foreign (glyco)lipids ${ }^{2}$ and has become an important target for study since the discovery that it is responsible for recognition of mycobacterial cord factor (trehalose dimycolate; TDM) ${ }^{3}$ and the synthetic adjuvant trehalose dibehenate (TDB) (Fig. 1a). ${ }^{4}$ Ligand binding to Mincle results in phosphorylation of the immunoreceptor tyrosine activation motif of $\mathrm{FCR}^{5,6}$ and activation of NF-KB via Card9-Bcl10-Malt1 signalosomes. ${ }^{7}$ Mincle is required for the classic granulomatous response to $\mathrm{TDM}^{3}$ and the adjuvant activity of TDB. ${ }^{4}$ TDB was developed as an optimized analogue of TDM, and both compounds induce strong Th1 and Th17 cellular immune responses. ${ }^{2}$ Mincle possesses an extracellular C-type lectin domain that appears to be the primary site for ligand binding. X-ray structures of human ${ }^{8}$ and bovine Mincle (the latter bound to trehalose and trehalose monobutyrate $)^{9}, 10$ revealed two monosaccharide binding subsites, with the primary site involving coordination of $\mathrm{O} 3$ and $\mathrm{O} 4$ to $\mathrm{Ca}^{2+}$, and a shallow hydrophobic groove that can accommodate lipid chains (Fig. 1b).

\footnotetext{
a. School of Chemistry and Bio21 Molecular Science and Biotechnology Institute, University of Melbourne, Parkville, Vic 3010.

b. Department of Molecular Immunology, Immunology Frontier Research Center, Osaka University, Osaka 565-0871, Japan.

Department of Molecular Immunology, Research Institute for Microbial Diseases, Osaka University, Osaka 565-0871, Japan

+ Footnotes relating to the title and/or authors should appear here.

Electronic Supplementary Information (ESI) available: [details of any supplementary information available should be included here]. See DOI: 10.1039/x0xx00000x
}

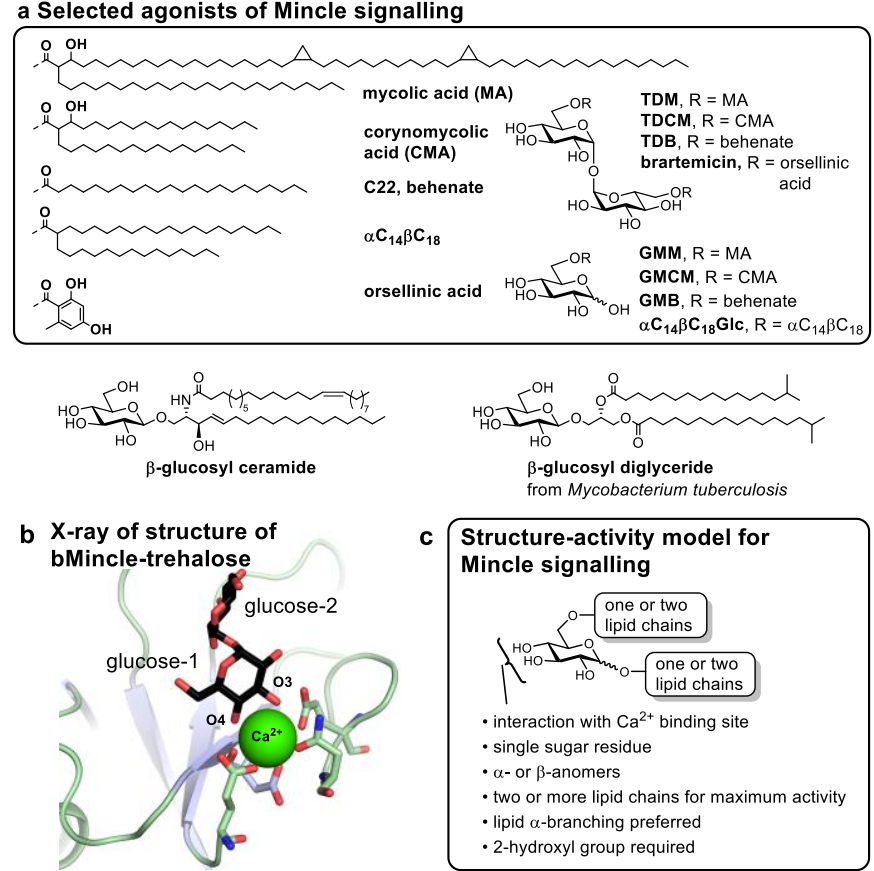

Fig. 1. a) Examples of potent agonists of Mincle signalling. b) X-ray structure of trehalose binding to the $\mathrm{Ca}^{2+}$ ion in the carbohydrate binding domain of Mincle (PDB 4KZV). c) Summary of structure-activity model for Mincle signalling.

A growing appreciation of the features necessary for agonism of Mincle signalling have emerged through identification of other naturally-occurring Mincle agonists, and through structure-activity relationship studies (reviewed in Refs ${ }^{2,11}$ ). Broadly, signalling through Mincle increases as lipid chain length increases for both mono- and diacyl-trehaloses, with activity maximized for a $\mathrm{C}_{22}$ (behenate) chain, ${ }^{12,13}$ or slightly shorter for a series of alkylated brartemicin analogues (Fig. 1a). ${ }^{14}$ A single glucose residue is sufficient for Mincle signalling: both glucose monomycolate $(\mathrm{GMM})^{15}$ and monocorynomycolate $(\mathrm{GMCM})^{16}$ are potent Mincle signalling 
agonists. Glucose 6-monobehenate $(\mathrm{GMB})^{16}$ is a weak Mincle agonist, yet glucose analogues bearing long, branched fatty acids linked off the 6 -position (such as $\alpha \mathrm{C}_{14} \beta \mathrm{C}_{18}$ ) $^{15}$ are potent agonists. Collectively, these results suggest that at least two lipid chains off a monosaccharide are necessary for optimum activity. Separately, various $\alpha$ - and $\beta$-glucosyl diacylglycerides ${ }^{17}$ ${ }^{19}$ and $\beta$-glucosyl ceramide ${ }^{20}$ can signal through Mincle, showing that the lipid group can be sited at the anomeric or C6-positions of glucose. Consistent with the requirement for at least two lipid chains for activity of monosaccharide glycolipids, a lyso $\beta$ glucosyl diacylglyceride did not signal through Mincle. ${ }^{18}$ Through studies of tetraacylated trehalose glycolipids it was concluded that a free 2 -hydroxyl group on a glucose residue was essential for activity. ${ }^{15}$

A good understanding now exists of structure-activity relationships for glucolipid signalling through Mincle (Fig. 1c). Decout and co-workers have argued that at least three of four key structural motifs (two sugars, two alkyl chains) must be present for Mincle agonism, and when combined with appropriate branching and length, the alkyl chains are more important than the second sugar unit. ${ }^{15} \mathrm{~A}$ separate study showed that branching at the $\beta$-position of a 6 - $O$-acyl-glucose analogue provided only weak Mincle agonism. ${ }^{21}$ Based on these results Decout and co-workers developed $\alpha \mathrm{C}_{14} \beta \mathrm{C}_{18} \mathrm{Glc}$, which possessed greater activity than TDB in a Mincle reporter assay. ${ }^{15}$ However, $\alpha \mathrm{C}_{14} \beta \mathrm{C}_{18} \mathrm{Glc}$ is comprised of complex mixture of 8 compounds: epimers at the $\alpha$-branched stereocentre of the lipid, the sugar hemiacetal that exists as both $\alpha$ - and $\beta$-anomers, as well as furanose and pyranose ring isomers; the contribution of each of these forms to activity is unclear.

In this work we report new, simplified agonists of Mincle signalling based on a $\beta$-glucoside template. In particular, we focussed on synthetic targets that can be easily prepared in just a few steps from commercial materials and which exist in a single isomeric form to simplify characterization, purification and interpretation of results. Cognizant of the potency of GMM and analogues as well as $\beta$-glucosyl diglycerides, we investigated compounds that possess lipophilic functionality at the 1- and 6-positions. Compounds of this general structure fit with the structure-activity relationships for Mincle signalling by possessing at least two alkyl chains, a single sugar residue and maintaining the free sugar 2-, 3- and 4-hydroxyl groups.

Alkyl glucosides are widely used in consumer products including cosmetics and soaps and both octyl and lauryl $\beta$ glucoside are commercially available reagents that could provide easy synthetic access to potential Mincle agonists. We avoided the use of protecting groups and instead performed direct acylation. Uronium-based peptide coupling reagents allow primary-selective acylation of simple glucosides through the choice of appropriate amine bases. ${ }^{22-24}$ We chose to install three representative straight chain esters: a short octanoyl $\left(C_{8}\right)$, medium palmitoyl $\left(C_{16}\right)$ and long behenoyl $\left(C_{22}\right)$ group (Scheme 1). Reaction of the corresponding acids with octyl $\beta$-glucoside afforded $\mathrm{C}_{8} \mathrm{GlcC}_{8} 3, \mathrm{C}_{16} \mathrm{GlcC}_{8} 4$ and $\mathrm{C}_{22} \mathrm{GlcC}_{8} 5$ in 61,33 and $47 \%$ yields, respectively. Similarly, the same acids and lauryl $\beta$ glucoside afforded $\mathrm{C}_{8} \mathrm{GlcC}_{12} 6, \mathrm{C}_{16} \mathrm{GlcC}_{12} 7$ and $\mathrm{C}_{22} \mathrm{GlcC}_{12} 8$ in 56, 46 and $47 \%$ yields, respectively.
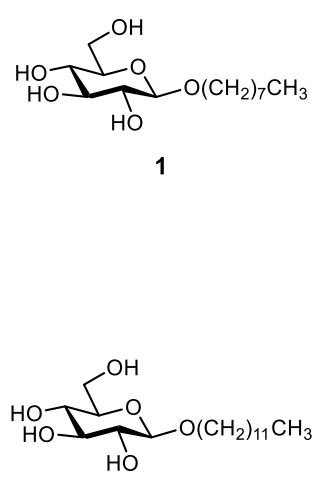

2 $a, b, c$ or d

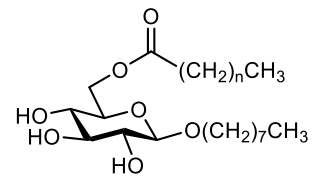

3, $\mathrm{R}=\mathrm{C}_{7} \mathrm{H}_{15}, 61 \%$

$4, \mathrm{R}=\mathrm{C}_{15} \mathrm{H}_{31}, 33 \%$

$5, \mathrm{R}=\mathrm{C}_{21} \mathrm{H}_{43}, 47 \%$

9, $\mathrm{R}=\mathrm{CH}\left[\left(\mathrm{CH}_{2}\right)_{13} \mathrm{CH}_{3}\right]_{2}$,

$15 \%$

$a, b, c$ or $d$

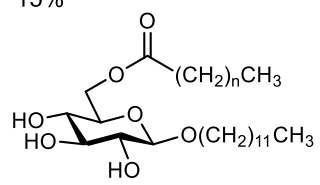

6, $\mathrm{R}=\mathrm{C}_{7} \mathrm{H}_{15}, 61 \%$

$7, \mathrm{R}=\mathrm{C}_{15} \mathrm{H}_{31}, 33 \%$

8, $\mathrm{R}=\mathrm{C}_{21} \mathrm{H}_{43}, 47 \%$

10, $\mathrm{R}=\mathrm{CH}\left[\left(\mathrm{CH}_{2}\right)_{13} \mathrm{CH}_{3}\right]_{2}$,

$12 \%$

Scheme 1. Synthesis of lauryl and octyl 6-O-acyl- $\beta$-D-glucopyranosides Reagents and conditions: a) octanoic acid, HBTU, pyr, b) palmitic acid, HBTU, pyr, c) behenic acid, $\mathrm{HBTU}$, pyr, d) $\mathrm{HO}_{2} \mathrm{CCH}\left[\left(\mathrm{CH}_{2}\right)_{15} \mathrm{CH}_{3}\right]_{2}, \mathrm{HBTU}$, pyr.

Racemic 2-tetradecyloctanoic acid was used by Decout et al. ${ }^{15}$ for preparation of $\alpha \mathrm{C}_{14} \beta \mathrm{C}_{18} \mathrm{Glc}$, and thus this compound is a mixture of isomers epimeric at the $\alpha$-position. We instead utilized commercially-available achiral 2-hexadecyloctanoic acid. Reaction of octyl and lauryl $\beta$-glucosides with this acid and HBTU in pyridine afforded the monoesters $\mathrm{C}_{18} \mathrm{C}_{16} \mathrm{GlcC}_{8} 9$ and $\mathrm{C}_{18} \mathrm{C}_{16} \mathrm{GlcC}_{12} 10$ in 15 and $12 \%$ yields, respectively. These yields were lower than that of the straight chain acids and may reflect reduced reactivity arising from the $\alpha$-branch.

Cholesterol can signal through Mincle, ${ }^{25}$ and is a lipophilic group that we speculated could occupy the lipid binding pocket. We synthesized 1-cholesteryloxyacetic acid $\mathbf{1 3}$ by reaction of ethyl diazoacetate ${ }^{26}$ with cholesterol promoted by $\mathrm{BF}_{3} . \mathrm{Et}_{2} \mathrm{O}$ in $\mathrm{CH}_{2} \mathrm{Cl}_{2}$ to afford 12 in $61 \%$ yield (Scheme 2). Saponification with $2 \mathrm{M} \mathrm{NaOH}$ in EtOH afforded 13. Reaction of octyl and lauryl $\beta$ glucosides with 13 and HBTU/pyridine afforded the monoesters CholGlcC $_{8} 14$ and CholGlcC 12 in 47 and $45 \%$ yields, respectively.

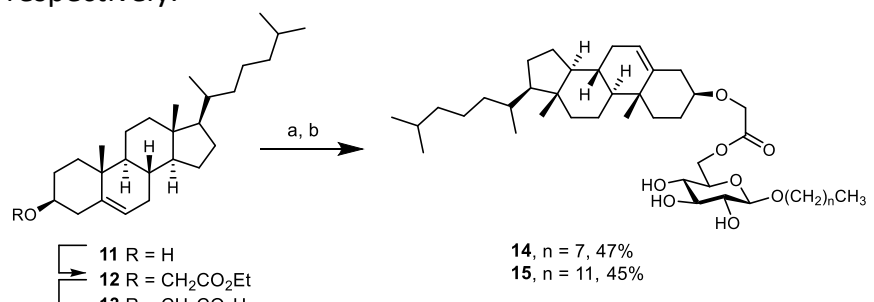

Scheme 2. Synthesis of lauryl and octyl 6-O-(cholesteryloxyacetyl)- $\beta$-Dglucopyranosides. Reagents and conditions: a) ethyl diazoacetate, $\mathrm{BF}_{3} . \mathrm{Et}_{2} \mathrm{O}$, $\mathrm{CH}_{2} \mathrm{Cl}_{2}$, b) $2 \mathrm{M} \mathrm{NaOH}$, EtOH, $\left.\mathrm{H}_{2} \mathrm{O}, \mathrm{c}\right) 1$ or 2, HBTU, pyr.

The panel of alkyl 6-O-acyl- $\beta$-D-glucopyranosides as well as the parent octyl and lauryl glucosides were investigated for their ability to induce signalling through Mincle. Assays were performed by culturing reporter cells expressing mouse and 
human Mincle on plate-bound glycolipids, and measurement of green fluorescent protein by flow cytometry to quantify the degree of Mincle signalling agonism. Octyl and lauryl $\beta$-Dglucopyranosides did not signal through Mincle, which is unsurprising as they are soluble detergents, and do not fulfil the expected structural criteria for Mincle agonists. All of the remaining compounds signalled to varying degrees through the two receptor orthologs.

For the straight-chain 6-O-acyl derivatives, a small increase in signalling potency was seen in changing from octyl to lauryl, except in the case of $\mathrm{C}_{22} \mathrm{GlcC}_{8}$ and $\mathrm{C}_{22} \mathrm{GlcC}_{12}$, where the potency was lower in the latter. Also, the intensity of signalling through the mouse and human orthologs was similar for most

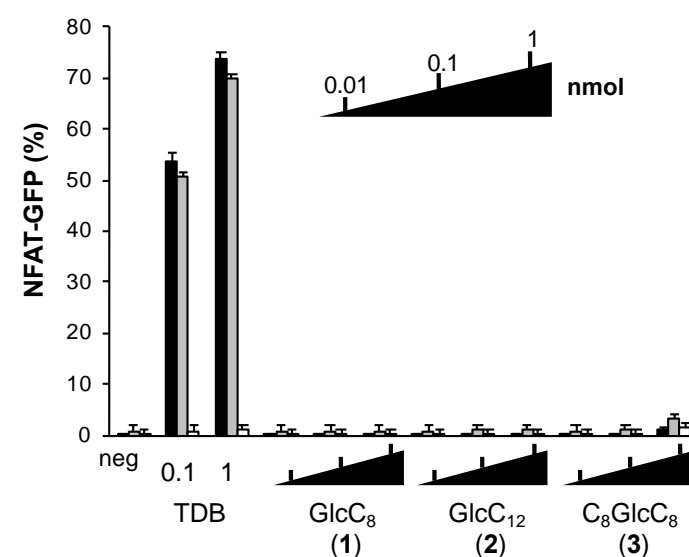

(1)
(2) analogues, except the $\mathrm{C}_{22}$ derivatives, where signalling through human Mincle was reduced relative to the mouse ortholog. Similarly, within the individual series of octyl or lauryl glycosides, an increase in 6-O-acyl chain length generally led to an increase in potency, with the exception of $\mathrm{C}_{22} \mathrm{GlcC}_{12}$, where the potency was reduced. In all cases, signalling potency was less than that of TDB. This trend in activity is reminiscent of the ability of trehalose mono-13 and diesters ${ }^{12}$ to activate macrophages, wherein the $\mathrm{C}_{22}$ compounds were more potent than the $\mathrm{C}_{26}$ analogues, and a homologous series of acyl glycerols, where potency peaked at $\mathrm{C}_{28}{ }^{27}$

Fig. 2 Agonism of Mincle signalling by unsubstituted octyl or lauryl $\beta$-D-glucopyranosides or straight-chain 6-O-acyl variants. NFAT-GFP reporter cells expressing either human Mincle/FcRy or mouse Mincle/FcR $\gamma$, as well as those expressing FcRy alone were tested for their reactivity to plate-bound TDB and analogues 18. Assays were performed in duplicate; the mean values and standard errors are shown. neg = isopropanol vehicle control.

We next evaluated the activities of the branched-chain 6-Oacyl $\beta$-D-glucopyranosides and the cholesteryloxyacetyl analogues (Fig. 3). The branched-chain analogues were more potent than any of the straight-chain derivatives, and again, little difference was seen between the octyl and lauryl glycosides. As for the $\mathrm{C}_{22}$ analogues, signalling intensity was lower for human Mincle than for mouse Mincle. The most potent signalling was seen for the cholesterol analogues, which provided signalling with similar intensities and potencies as TDB and comprise the most potent agonists identified in this study.

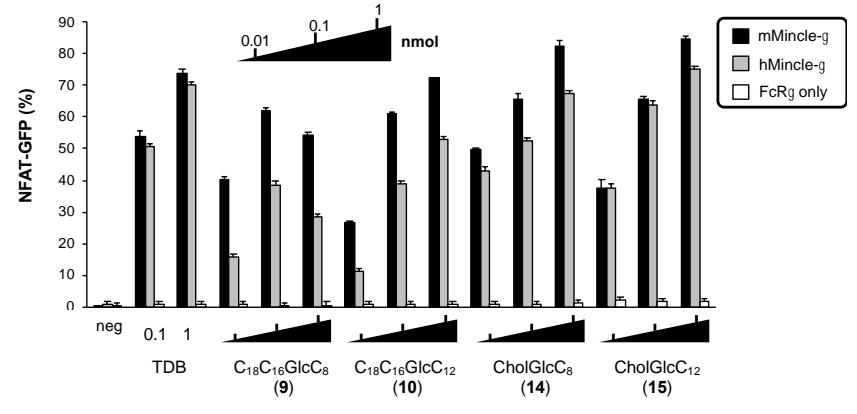

Fig. 3 Agonism of Mincle signalling by octyl or lauryl 6-O-acyl- $\beta$-Dglucopyranosides bearing a branched lipid chain or a cholesteryloxyacetyl group. NFAT-GFP reporter cells expressing either human Mincle/FcRy or mouse Mincle/FcRy, as well as those expressing FcRy alone were tested for their reactivity to plate-bound TDB and analogues 9, 10, 14 and 15. Assays were performed in duplicate; the mean values and standard errors are shown. neg = isopropanol vehicle control. Results for TDB and neg were obtained in the same experiment as for Fig. 2.

In conclusion this work reports development of simple alkyl glucosides as highly potent Mincle agonists based on emerging structure-activity relationships. Long-chain lipidic esters at 06 provided potent agonists of Mincle signalling, with even greater activity seen for an $\alpha$-branched fatty acyl chain. These results are broadly in concordance with the structure-activity relationship articulated by Decout et al. ${ }^{15}$ but extend that work by showing that a lipid chain at $\mathrm{C} 1$ can contribute to potency and simplify their structures by both blocking the anomeric position and locking its stereochemistry. Our results also show that alkyl $\beta$-glucosides esterified with a cholesteryl group at 06 are potent Mincle antagonists. Cholesterol, in its crystalline insoluble form, signals through human, but not rodent Mincle. ${ }^{25}$ Signalling by cholesterol involves interaction with the cholesterol recognition site (CRAC) of human Mincle, which is absent in rodent Mincle, and alkylation of cholesterol leads to loss of ability to signal through Mincle. ${ }^{25}$ As our analogues are alkylated cholesterol derivatives, and signal through both mouse and human Mincle, it seems unlikely that the cholesteryl 
moiety binds at the CRAC site of Mincle and is likely simply a surrogate for a lipid group.

This work builds on related efforts exploring structure activity relationships of acyl-hexoses (as analogues of glucose monomycolate), ${ }^{15,16}$ glycosyloxy-stearates (as analogues of the mannosyloxymannitol glycolipid from Malassezia pachydermatis), ${ }^{28}$ glycerolipids (as analogues of glycerol monomycolate), 27, 29 mono-13 and diacyl trehaloses, ${ }^{12,} 30$ and lipidated diaroyltrehaloses (as analogues of brartemicin). ${ }^{14,} 31$ The present work is notable for the simplicity of the resulting agonists and the fact that unlike acyl-hexoses they exist as single stereoisomers.

We thank the Australian Research Council (DP160100597) and AMED (JP19gm0910010, JP19ak0101070 and JP19fk0108075) for grant support.

\section{Conflicts of interest}

There are no conflicts to declare.

\section{Notes and references}

1

3 E. Ishikawa, T. Ishikawa, Y. S. Morita, K. Toyonaga, H. Yamada, O. Takeuchi, T. Kinoshita, S. Akira, Y. Yoshikai and S. Yamasaki, J. Exp. Med., 2009, 206, 2879. H. Schoenen, B. Bodendorfer, K. Hitchens, S. Manzanero, K. Werninghaus, F. Nimmerjahn, E. M. Agger, S. Stenger, P. Andersen, J. Ruland, G. D. Brown, C. Wells and R. Lang, J. Immunol., 2010, 184, 2756.

S. Yamasaki, E. Ishikawa, M. Sakuma, H. Hara, K. Ogata and T. Saito, Nat. Immunol., 2008, 9, 1179. M. R. Daws, Eur. J. Immunol., 2013, 43, 3167.

K. Werninghaus, A. Babiak, O. Gross, C. Holscher, H. Dietrich, E. M. Agger, J. Mages, A. Mocsai, H. Schoenen, K. 2009, 206, 89.

A. Furukawa, J. Kamishikiryo, D. Mori, K. Toyonaga, Y. K. Maenaka, Proc. Natl. Acad. Sci. USA, 2013, 110, 17438.

H. Feinberg, S. A. Jegouzo, T. J. Rowntree, Y. Guan, M. A. Brash, M. E. Taylor, W. I. Weis and K. Drickamer, J. Biol. Chem., 2013, 288, 28457.

H. Feinberg, N. D. Rambaruth, S. A. Jegouzo, K. M. Jacobsen, R. Djurhuus, T. B. Poulsen, W. I. Weis, M. E. Taylor and K. Drickamer, J. Biol. Chem., 2016, 291, 21222. X. Lu, M. Nagata and S. Yamasaki, Int. Immunol., 2018, 30, 233. 2011, 12, 2572.

B. L. Stocker, A. A. Khan, S. H. Chee, F. Kamena and M. S.
A. Lobato-Pascual, P. C. Saether, S. Fossum, E. Dissen and Finger, F. Nimmerjahn, G. D. Brown, C. Kirschning, A. Heit, P. Andersen, H. Wagner, J. Ruland and R. Lang, J. Exp. Med., Okabe, A. Toji, R. Kanda, Y. Miyake, T. Ose, S. Yamasaki and

A. A. Khan, S. H. Chee, R. J. McLaughlin, J. L. Harper, F. Kamena, M. S. M. Timmer and B. L. Stocker, Chembiochem, Timmer, Chembiochem, 2014, 15, 382.
14

15

18

A. J. Foster, M. Nagata, X. Lu, A. T. Lynch, Z. Omahdi, E. Ishikawa, S. Yamasaki, M. S. M. Timmer and B. L. Stocker, J. Med. Chem., 2018, 61, 1045.

A. Decout, S. Silva-Gomes, D. Drocourt, S. Barbe, I. Andre, F. J. Cueto, T. Lioux, D. Sancho, E. Perouzel, A. Vercellone, J. Prandi, M. Gilleron, G. Tiraby and J. Nigou, Proc. Natl. Acad. Sci. USA, 2017, 114, 2675.

P. L. van der Peet, C. Gunawan, S. Torigoe, S. Yamasaki and S. J. Williams, Chem. Commun., 2015, 51, 5100.

S. Shah, M. Nagata, S. Yamasaki and S. J. Williams, Chem. Commun., 2016, 52, 10902.

M. B. Richardson, S. Torigoe, S. Yamasaki and S. J. Williams, Chem. Commun., 2015, 51, 15027.

F. Behler-Janbeck, T. Takano, R. Maus, J. Stolper, D. Jonigk, M. Tort Tarres, T. Fuehner, A. Prasse, T. Welte, M. S. Timmer, B. L. Stocker, Y. Nakanishi, T. Miyamoto, S. Yamasaki and U. A. Maus, PLoS Pathog., 2016, 12, e1006038.

M. Nagata, Y. Izumi, E. Ishikawa, R. Kiyotake, R. Doi, S. Iwai, Z. Omahdi, T. Yamaji, T. Miyamoto, T. Bamba and S. Yamasaki, Proc. Natl. Acad. Sci. USA, 2017, 114, E3285.

21 P. L. van der Peet, M. Nagata, S. Shah, J. M. White, S. Yamasaki and S. J. Williams, Org. Biomol. Chem., 2016, 14, 9267.

Z. Hakki, B. Cao, A. M. Heskes, J. Q. Goodger, I. E. Woodrow and S. J. Williams, Carbohydr. Res., 2010, 345, 2079. J. D. Twibanire and T. B. Grindley, Org. Lett., 2011, 13 2988.

N. K. Paul, J. D. Twibanire and T. B. Grindley, J. Org. Chem., 2013, 78, 363.

25 R. Kiyotake, M. Oh-Hora, E. Ishikawa, T. Miyamoto, T. Ishibashi and S. Yamasaki, J. Biol. Chem., 2015, 290, 25322. D. Lafont, P. Boullanger and A. Gambetta, J. Labelled Compds. Radiopharmaceut., 2012, 55, 88.

T. Matsumaru, R. Ikeno, Y. Shuchi, T. Iwamatsu, T. Tadokoro, S. Yamasaki, Y. Fujimoto, A. Furukawa and K. Maenaka, Chem. Commun., 2019, 55, 711.

L. Van Huy, C. Tanaka, T. Imai, S. Yamasaki and T. Miyamoto, ACS Med. Chem. Lett., 2019, 10, 44.

29 A. Khan, C. D. Braganza, K. Kodar, M. S. M. Timmer and B. L. Stocker, Org. Biomol. Chem., 2020, DOI: 10.1039/С9OB02302J.

30 J. H. Bird, A. A. Khan, N. Nishimura, S. Yamasaki, M. S. M. Timmer and B. L. Stocker, J. Org. Chem., 2018, 83, 7593.

31 A. J. Foster, K. Kodar, M. S. M. Timmer and B. L. Stocker, Org. Biomol. Chem., 2020, DOI: 10.1039/C9OB02397F. 